Article

\title{
Hexachlorocyclotriphosphazene (HCCP)-Mediated Direct Formation of Thioethers and Ethers from Quinazolin-4(3H)-ones
}

\author{
Baoxiang Hu ${ }^{1}$, Xiaochu Zhang ${ }^{1}$, Lili Sheng ${ }^{1}$, Ming Guo ${ }^{2}$, Zhenlu Shen ${ }^{1{ }^{1} *}$, Xinquan Hu ${ }^{1}$, \\ Nan Sun ${ }^{1}$ and Weimin Mo ${ }^{1, *}$ \\ 1 State Key Laboratory Breeding Base of Green Chemistry-Synthesis Technology, \\ College of Chemical Engineering and Materials Science, Zhejiang University of Technology, \\ Hangzhou 310014, China \\ 2 Department of Chemistry, Zhejiang Agriculture \& Forestry University, Lin'An 311300, China \\ * Authors to whom correspondence should be addressed; E-Mails: zhenlushen@zjut.edu.cn (Z.S.); \\ mowm@zjut.edu.cn (W.M.); Tel.: +86-571-8832-0416 (Z.S.); Fax: +86-571-8832-0103 (Z.S.).
}

Received: 25 March 2013; in revised form: 12 May 2013 / Accepted: 13 May 2013 /

Published: 15 May 2013

\begin{abstract}
A hexachlorocyclotriphosphazene (HCCP)-mediated direct formation of quinazoline (thio)ethers from quinazolin-4(3H)-ones has been developed. Treatment of quinazolin-4(3H)-ones with HCCP, diisopropylethylamine (DIPEA), and thiophenols resulted in formation of the corresponding 4-arylthioquinazoline derivatives in moderate to excellent yields. This method has also been utilized to prepare 4-aryloxyquinazoline and 4-alkoxyquinazoline derivatives using phenols and sodium alkoxides as the nucleophiles.
\end{abstract}

Keywords: quinazolin-4(3H)-ones; hexachlorocyclotriphosphazene; 4-arylthioquinazolines; 4-aryloxyquinazolines; 4-alkoxyquinazolines

\section{Introduction}

Quinazoline derivatives are an important class of nitrogen-containing heterocycles. They have attracted interest in the past because of their varied biological activities, such as anticonvulsant, antihypertensive, vasodilator, antiinflammatory, antibiosis, fibrinogen receptor antagonistic and nanomolar Hedgehog-antagonistic properties [1-8]. Among the family of quinazolines, quinazoline (thio)ethers, including 4-arylthioquinazolines and 4-aryloxyquinazolines, have received considerable interest because of their potential pharmacological activity [9-16]. 
Generally, 4-arylthioquinazolines and 4-aryloxyquinazolines are obtained through $\mathrm{S}_{\mathrm{N}} \mathrm{Ar}$ substitution of electron-deficient 4-chloroquinazolines with the appropriate thiophenols or phenols in the presence of a base [9-19]. The common method for preparation of 4-chloroquinazolines is the chlorination of corresponding quinazolin-4(3H)-ones. The chlorination reagents used include $\mathrm{SOCl}_{2}, \mathrm{POCl}_{3}, \mathrm{PCl}_{5}$ or their combinations, and the chlorination reactions are usually performed under harsh conditions. However, these chlorination reagents are not environmentally friendly, and some sensitive functional groups may be destroyed under the chlorination conditions. In addition, many 4-chloroquinazoline derivatives are moisture sensitive, thus special treatments are required for their purification and storage.

In order to avoid the use of chlorination reagents and an individual activation step, an in situ activation of quinazolin-4(3H)-ones is highly desirable. Phosphonium compounds, such as benzotriazol1-yloxytris(dimethylamino)phosphonium hexafluorophosphate (BOP), have been developed to activate quinazolin-4(3H)-ones and successfully employed in the synthesis of 4-aminoquinazolines, 4-arylthioquinazolines and 4-aryloxyquinazolines using amine, thiophenol and phenol nucleophiles, respectively [20,21]. However, BOP and other phosphonium reagents are expensive, moreover, utilization of BOP generates as an end product HMPA, a highly carcinogenic chemical.

As a part of our research program focused on quinazoline chemistry [22,23], we have recently, reported that hexachlorocyclotriphosphazene $\left(\mathrm{Cl}_{6} \mathrm{~N}_{3} \mathrm{P}_{3}, \mathrm{HCCP}\right)$ can be used as an inexpensive and readily available activating reagent in the direct amination of quinazolin-4(3H)-ones (1) [24]. In the reaction, quinazolin-4(3H)-ones are activated in situ with HCCP to generate the highly reactive phosphonium intermediate $\mathbf{2}$, then amines can nucleophilically attack $\mathbf{2}$ to form 4 -aminoquinazolines. Thus, we speculated that other nucleophiles, such as thiophenol and phenol ones, might attack 2 to form the corresponding products. Herein, we report a HCCP-mediated, single-pot, facile synthesis of thioethers and ethers from quinazolin-4(3H)-ones in moderate to excellent yields (Scheme 1).

Scheme 1. One-pot synthesis of quinazoline (thio)ethers from quinazolin-4(3H)-ones mediated by HCCP.

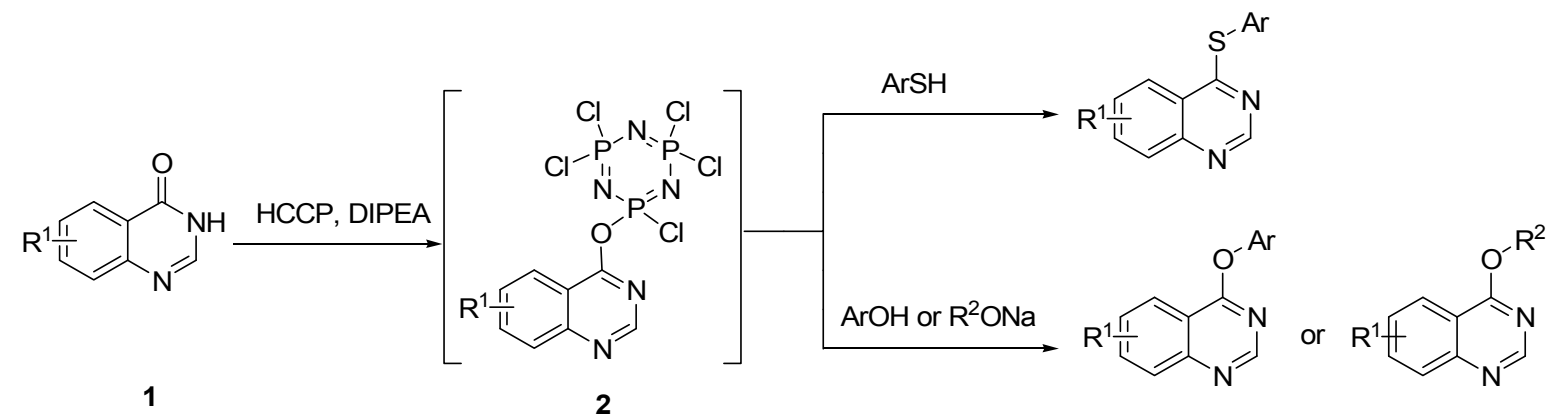

\section{Results and Discussion}

Initially, the reaction of quinazolin-4(3H)-one (1a) and thiophenol was investigated as the model reaction to establish the feasibility of the strategy and to optimize the reaction conditions. The effects of solvent, base, temperature and HCCP loading, etc., were evaluated for this model reaction, and the results are summarized in Table 1. 
Table 1. Optimization of reaction conditions ${ }^{\mathrm{a}}$.

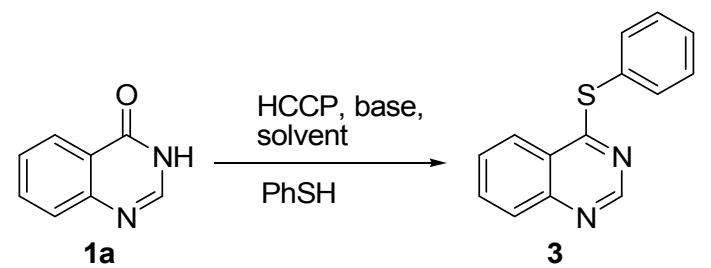

\begin{tabular}{ccccc}
\hline Entry & Base & Solvent & Temperature $\left({ }^{\circ} \mathbf{C}\right)$ & Yield $(\%))^{\mathbf{b}}$ \\
\hline 1 & TEA & $\mathrm{MeCN}$ & 45 & 48 \\
2 & $\mathrm{~K}_{2} \mathrm{CO}_{3}$ & $\mathrm{MeCN}$ & 45 & 32 \\
3 & $\mathrm{Cs}_{2} \mathrm{CO}_{3}$ & $\mathrm{MeCN}$ & 45 & 69 \\
4 & $\mathrm{DIPEA}$ & $\mathrm{MeCN}$ & 45 & 81 \\
5 & $\mathrm{DIPEA}$ & $\mathrm{THF}$ & 45 & 12 \\
6 & $\mathrm{DIPEA}$ & $\mathrm{DMF}$ & 45 & 23 \\
7 & DIPEA & $\mathrm{CH}_{2} \mathrm{Cl}$ & 45 & 7 \\
8 & DIPEA & $\mathrm{MeCN}$ & 45 & $76^{\mathrm{c}}$ \\
9 & DIPEA & $\mathrm{MeCN}$ & 25 & 68 \\
10 & DIPEA & $\mathrm{MeCN}$ & 65 & 55 \\
11 & DIPEA & $\mathrm{MeCN}$ & 45 & $79^{\mathrm{d}}$ \\
12 & DIPEA & $\mathrm{MeCN}$ & 45 & $71^{\mathrm{e}}$ \\
13 & DIPEA & $\mathrm{MeCN}$ & 45 & $81^{\mathrm{f}}$ \\
14 & DIPEA & $\mathrm{MeCN}$ & 45 & $70^{\mathrm{g}}$ \\
\hline
\end{tabular}

${ }^{a}$ Conditions: 1a $(0.5 \mathrm{mmol}), \mathrm{HCCP}$ (1.1 equiv.), base $(5.0$ equiv.), solvent $(5 \mathrm{~mL})$, rt, activation time $(1 \mathrm{~h})$, then thiophenol (6.0 equiv.), $45{ }^{\circ} \mathrm{C}, 23 \mathrm{~h} ;{ }^{\mathrm{b}}$ Isolated yield; ${ }^{\mathrm{c}} \mathrm{HCCP}$ (1.0 equiv.); ${ }^{\mathrm{d}}$ Thiophenol (5.0 equiv.);

e Thiophenol (4.0 equiv.); ${ }^{\mathrm{f}}$ DIPEA (6.0 equiv.); ${ }^{\mathrm{g}}$ DIPEA (4.0 equiv.).

Compared with triethylamine (TEA), $\mathrm{K}_{2} \mathrm{CO}_{3}$ and $\mathrm{Cs}_{2} \mathrm{CO}_{3}$ (entries 1-3), diisopropylethylamine (DIPEA) was found to be the most effective in the model reaction of $\mathbf{1 a}$ and thiophenol (entry 4). The results in Table 1 show that the solvent can greatly affect the reaction, and acetonitrile led to much better yield of product 3 than tetrahydrofuran, $N, N$-dimethylformamide or dichloromethane (entries 4-7). According to Scheme 1, 1.0 equiv. HCCP loading was enough, but we found that increasing the HCCP loading from 1.0 to 1.1 equiv. resulted in 5\% yield increases (entry 8 versus entry 4 ). After in situ activation of $1 \mathrm{a}$ with HCCP at room temperature for $1 \mathrm{~h}$, the $\mathrm{S}_{\mathrm{N}} \mathrm{Ar}$ substitution of phosphonium intermediate 2a $\left(\mathrm{R}^{1}=\mathrm{H}\right)$ with thiophenol was performed at $45{ }^{\circ} \mathrm{C}$. At both lower and higher temperature, the yield of 3 decreased (entries 9 and 10). Like the HCCP-mediated direct amination of quinazolin-4(3H)-ones with amines [24], when the thiophenol nucleophile attacks 2 a, two competitive $\mathrm{S}_{\mathrm{N}} \mathrm{Ar}$ substitutions were possible, either on the $\mathrm{C}-\mathrm{O}$ bond or $\mathrm{P}-\mathrm{Cl}$ bond. Thus, five to six equiv. of thiophenol and DIPEA were needed in this reaction. It was found variation of the amount of thiophenol or DPIEA from 6.0 equiv. to 5.0 equiv. did not significantly change the results (entry 4 versus entries 11 and 13), whereas upon decreasing the amount of thiophenol or DPIEA to 4.0 equiv., the yield of 3 was obviously decreased (entries 12 and 14). On the basis of these experimental data, the optimal reaction conditions were: 1.1 equiv. of HCCP, 5.0 equiv. of DIPEA, 5.0 equiv. of thiophenol, $45{ }^{\circ} \mathrm{C}$ of reaction temperature and acetonitrile as the reaction solvent, and these were employed in the following studies. 
The results of HCCP-mediated direct formation of thioethers (4-arylthioquinazolines) from $\mathbf{1}$ and thiophenols are summarized in Table 2. Different substituted thiophenols smoothly reacted with 1a to afford the desired 4-arylthioquinazolines in moderate to excellent yields (entries 1-9). When $o, m$, and $p$-methoxythiophenols were used as the nucleophiles, $o$-methoxythiophenol gave the highest product yield, while $m$-methoxythiophenol showed the lowest reactivity (entries 4-6). It is noteworthy that chlorothiophenols, which usually exhibit lower nucleophilicity than methoxythiophenols, could conveniently undergo the transformation and gave excellent product yields in this HCCP-mediated direct formation of 4-arylthioquinazolines (entries 7 and 9). The reactions between thiophenol and a variety of substituted quinazolin-4(3H)-ones were also investigated. An array of substituted quinazolin-4(3H)-ones were suitable for this HCCP-mediated formation of thioethers and gave the desired 4-arylthioquinazolines in moderate to good yields (entries 1, 10-15). When 5-methylquinazolin-4(3H)-one (1) $)$ and 5-chloroquinazolin-4(3H)-one (1e) were used as the substrates, activation time should be prolonged (entries 10 and 13).

Table 2. HCCP-mediated formation of quinazoline thioethers from quinazolin-4(3H)-ones ${ }^{\text {a }}$.

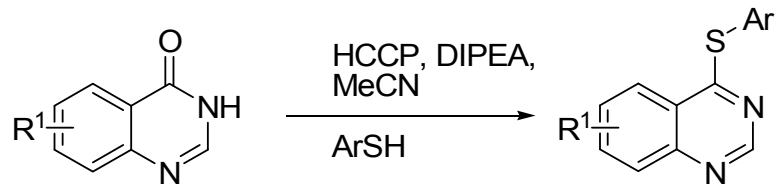

1 3-17

Entry Quinazolin-4(3H)-one Yield (\%)


Table 2. Cont.

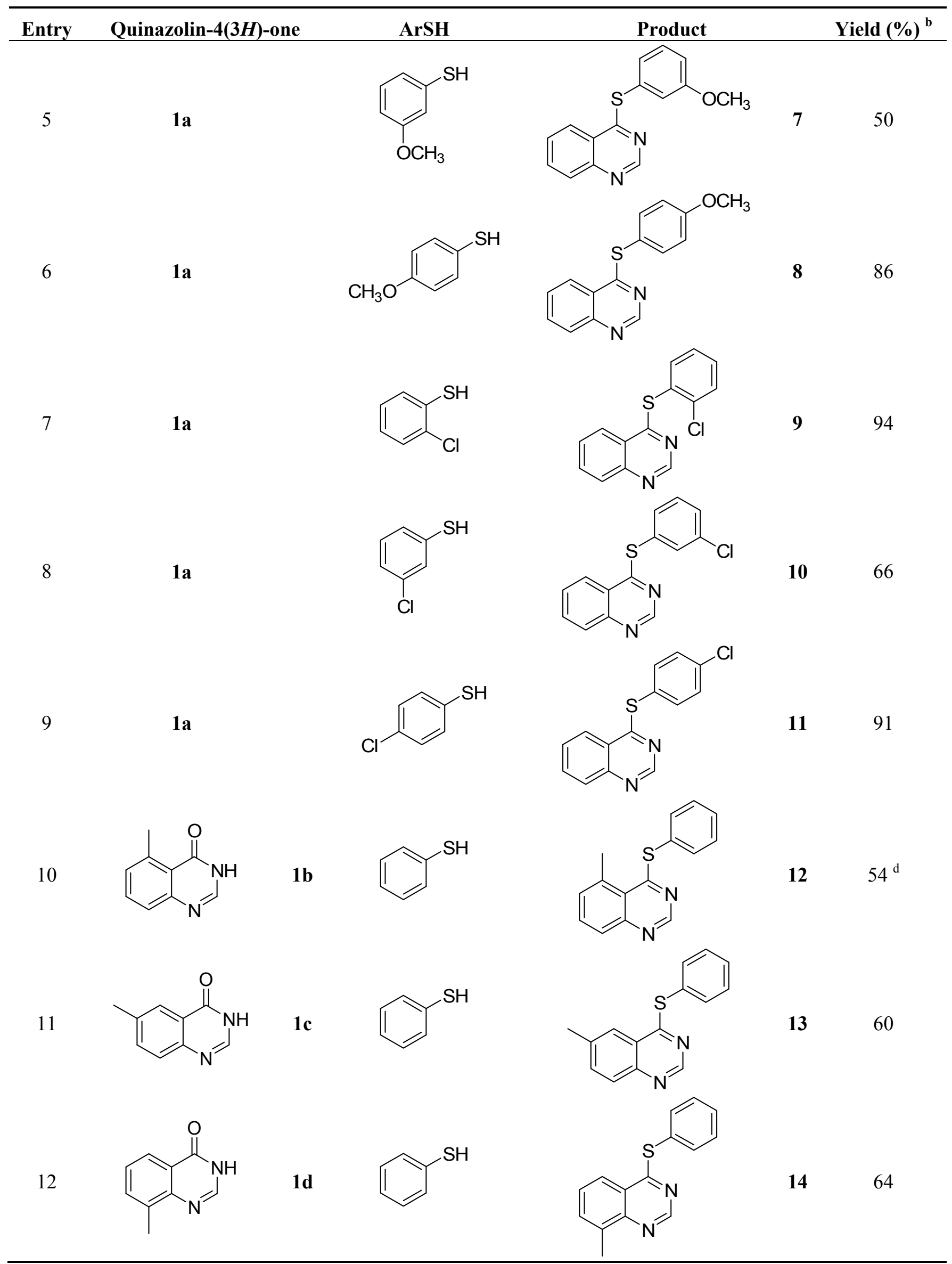


Table 2. Cont.

Entry Quinazolin-4(3H)-one Yield (\%)

${ }^{\mathrm{a}}$ Reagents and Conditions: 1 ( $\left.0.5 \mathrm{mmol}\right), \mathrm{HCCP}$ (1.1 equiv.), DIPEA (5.0 equiv.), MeCN ( $\left.5 \mathrm{~mL}\right), \mathrm{rt}$, activation time $(1 \mathrm{~h})$, then thiophenols $\left(5.0\right.$ equiv.), $45^{\circ} \mathrm{C}, 23 \mathrm{~h} ;{ }^{b}$ Isolated yield; ${ }^{\mathrm{c}} m$ - $\mathrm{CH}_{3} \mathrm{PhSH}$ (6.0 equiv.); reaction time (48 h); ${ }^{\mathrm{d}}$ Activation time $(20 \mathrm{~h})$.

The method was further extended to the synthesis of 4-aryloxyquinazolines through the reaction of 1 and phenols under the same reaction conditions (Table 3). Different phenols (phenol, $m$-methylphenol, $o$-chlorophenol and $p$-chlorophenol) could react with 1a and afforded the corresponding products 18-21 in moderate to good yields (entry 1-5). There is no significant electronic effect of substituents for products. Substituted quinazolin-4(3H)-ones were also used as the substrates to react with phenol. 6-Chloroquinazolin-4(3H)-one (1f) and 7-chloroquinazolin-4(3H)-one (1g) gave products 14 and $\mathbf{1 5}$ in $70 \%$ yield (entries 6 and 7), whereas in the case of 5-methylquinazolin-4(3H)-one (1b), the product 12 was obtained in 54\% yield (entry 5). Alcohols were also used as the nucleophiles in this reaction. Unfortunately, no desired 4-alkoxyquinazolines were formed. It might be due to that the nucleophilicity of alcohols was too weak to undego $S_{N} A r$ substitution. Thus, sodium alkoxides were further utilized as the nucleophiles in this reaction to give the corresponding 4-alkoxyquinazolines (Table 3). When 1a, 8-methylquinazolin-4(3H)-one (1d) and $\mathbf{1 g}$ reacted with sodium ethoxide for $3 \mathrm{~h}$, the yields of products 4-ethoxyquinazolines were $54 \%, 64 \%$ and $67 \%$, respectively (entries 8,10 and 11 ), while $1 \mathrm{~b}$ gave only $33 \%$ product yield (entry 9). 4-Propoxyquinazoline (29) could be obtained in $48 \%$ yield in the reaction between $1 \mathbf{a}$ and sodium propoxide (entry 12).

Table 3. HCCP-mediated formation of quinazoline ethers from quinazolin-4(3H)-ones ${ }^{\mathrm{a}}$.<smiles>O=c1[nH]cnc2cc[R1]cc12</smiles>

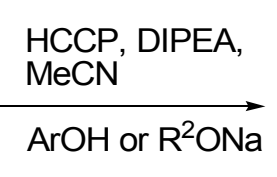

ArOH or $\mathrm{R}^{2} \mathrm{ONa}$<smiles>[Al]Oc1ncnc2cc[R11]cc12</smiles>

18-24<smiles>[R]Oc1ncnc2c1C=C[R1]C=C2</smiles> 
Table 3. Cont.

\begin{tabular}{|c|c|c|c|c|c|}
\hline Entry & Quinazolin-4(3H)-one & ArOH or RONa & Product & & Yield (\%) ${ }^{b}$ \\
\hline 1 & $\mathbf{1 a}$ & & & 18 & 75 \\
\hline 2 & $1 \mathbf{a}$ & & & 19 & 52 \\
\hline 3 & $1 \mathbf{a}$ & & & 20 & 73 \\
\hline 4 & $1 \mathbf{a}$ & & & 21 & 51 \\
\hline 5 & $1 \mathrm{~b}$ & & & 22 & $53^{\mathrm{c}}$ \\
\hline 6 & 1f & & & 23 & 70 \\
\hline 7 & $1 g$ & & & 24 & 70 \\
\hline 8 & $\mathbf{1 a}$ & $\mathrm{CH}_{3} \mathrm{CH}_{2} \mathrm{ONa}$ & & 25 & 54 \\
\hline
\end{tabular}


Table 3. Cont.

\begin{tabular}{|c|c|c|c|c|c|}
\hline Entry & Quinazolin-4(3H)-one & ArOH or RONa & Product & & Yield $(\%)^{b}$ \\
\hline 9 & $1 \mathrm{~b}$ & $\mathrm{CH}_{3} \mathrm{CH}_{2} \mathrm{ONa}$ & & 26 & $33^{\mathrm{c}}$ \\
\hline 10 & 1d & $\mathrm{CH}_{3} \mathrm{CH}_{2} \mathrm{ONa}$ & & 27 & 67 \\
\hline 11 & $1 \mathrm{~g}$ & $\mathrm{CH}_{3} \mathrm{CH}_{2} \mathrm{ONa}$ & & 28 & 64 \\
\hline 12 & $1 \mathbf{a}$ & $\mathrm{CH}_{3} \mathrm{CH}_{2} \mathrm{CH}_{2} \mathrm{ONa}$ & & 29 & 48 \\
\hline
\end{tabular}

${ }^{\mathrm{a}}$ Reagents and Conditions: 1 (0.5 mmol), HCCP (1.1 equiv.), DIPEA (5.0 equiv.), MeCN (5 mL), rt, activation time $(1 \mathrm{~h})$, then phenols $\left(5.0\right.$ equiv.), $45{ }^{\circ} \mathrm{C}, 23 \mathrm{~h}$; or $\mathrm{R}^{2} \mathrm{ONa}\left(5.0\right.$ equiv.), $45{ }^{\circ} \mathrm{C}, 3 \mathrm{~h}$; ${ }^{\mathrm{b}}$ Isolated yield; ${ }^{c}$ Activation time $(20 \mathrm{~h})$.

\section{Experimental}

\subsection{General}

${ }^{1} \mathrm{H}-\mathrm{NMR}(500 \mathrm{MHz})$ and ${ }^{13} \mathrm{C}-\mathrm{NMR}(125 \mathrm{MHz})$ spectra were obtained on a Bruker Avance III spectrometer. $\mathrm{CDCl}_{3}$ and DMSO- $d_{6}$ were used as the solvent with tetramethylsilane (TMS) as the internal standard. Low and high resolution mass spectra were recorded in the ESI mode on an Agilent 6210 LC/TOF mass spectrometer. Melting points were measured using XRL-1 melting point instrument and are uncorrected. Quinazolin-4(3H)-ones were synthesized from anthranilic acids and formamidine acetate, and their structures were confirmed by MS, ${ }^{1} \mathrm{H}-\mathrm{NMR}$, and ${ }^{13} \mathrm{C}-\mathrm{NMR}$. Other reagents were purchased from supplier and used without any further treatment.

\subsection{General Procedure for HCCP-Mediated Formation of Thioethers and Ethers from}

Quinazolin-4(3H)-ones

Quinazolin-4(3H)-ones (1, $0.5 \mathrm{mmol})$, HCCP (171.2 mg, $0.55 \mathrm{mmol}, 1.1$ equiv.), DIPEA (323.8 mg, $2.5 \mathrm{mmol}, 5$ equiv.), and $\mathrm{CH}_{3} \mathrm{CN}(5 \mathrm{~mL})$ were added to a nitrogen purged vial. The reaction mixture was stirred at room temperature for $1 \mathrm{~h}$ as activation time. Then nucleophile $(2.5 \mathrm{mmol}, 5$ equiv. was added, and the reaction mixture was stirred at $45{ }^{\circ} \mathrm{C}$ for $23 \mathrm{~h}$. The reaction mixture was partially concentrated under reduced pressure. The crude product was separated on a silica gel plate with ethyl acetate-hexane (1:10 or 1:5) as eluent. Then the area corresponding to the product was scraped off the 
TLC plate, and extracted with dichloromethane. The extract was concentrated to afford the corresponding products (3-29).

4-(Phenylthio)quinazoline (3). White solid (79\% yield); mp $109-110{ }^{\circ} \mathrm{C} ;{ }^{1} \mathrm{H}-\mathrm{NMR}$ (DMSO-d6) $\delta 8.84$ (s, 1H), 8.27 (d, $J=8.5 \mathrm{~Hz}, 1 \mathrm{H}), 8.07-8.04(\mathrm{~m}, 1 \mathrm{H}), 8.00-7.99(\mathrm{~m}, 1 \mathrm{H}), 7.83-7.79(\mathrm{~m}, 1 \mathrm{H}), 7.68-7.66$ $(\mathrm{m}, 2 \mathrm{H}), 7.56-7.54(\mathrm{~m}, 3 \mathrm{H}) ;{ }^{13} \mathrm{C}-\mathrm{NMR}$ (DMSO-d6) $\delta 170.3,153.5,147.8,135.8,134.6,129.9,129.6$, 128.5, 128.4, 126.5, 123.6, 122.3; HRMS (ESI), $m / z, 239.0644\left[\mathrm{MH}^{+}\right]$, calcd for $\mathrm{C}_{14} \mathrm{H}_{11} \mathrm{~N}_{2} \mathrm{~S}, 239.0643$.

4-(m-Tolylthio)quinazoline (4). White solid (64\% yield); mp 58-61 ${ }^{\circ} \mathrm{C} ;{ }^{1} \mathrm{H}-\mathrm{NMR}$ (DMSO-d6) $\delta 8.83$ (s, $1 \mathrm{H}), 8.25(\mathrm{~d}, J=8.0 \mathrm{~Hz}, 1 \mathrm{H}), 8.05-8.02(\mathrm{~m}, 1 \mathrm{H}), 7.99-7.97(\mathrm{~m}, 1 \mathrm{H}), 7.81-7.77(\mathrm{~m}, 1 \mathrm{H}), 7.48-7.41(\mathrm{~m}$, $3 \mathrm{H}), 7.36-7.35(\mathrm{~m}, 1 \mathrm{H}), 2.37(\mathrm{~s}, 3 \mathrm{H}) ;{ }^{13} \mathrm{C}-\mathrm{NMR}$ (DMSO-d6) $\delta 170.5,153.5,147.8,139.0,136.0,134.6$, 132.9, 130.6, 129.4, 128.5, 128.3, 126.2, 123.6, 122.4, 20.7; HRMS (ESI), $m / z, 253.0795\left[\mathrm{MH}^{+}\right]$, calcd for $\mathrm{C}_{15} \mathrm{H}_{13} \mathrm{~N}_{2} \mathrm{~S}, 253.0799$.

4-(p-Tolylthio)quinazoline (5) White solid (69\% yield); mp 104-105 ${ }^{\circ} \mathrm{C} ;{ }^{1} \mathrm{H}-\mathrm{NMR}$ (DMSO-d6) $\delta 8.81$ $(\mathrm{s}, 1 \mathrm{H}), 8.19(\mathrm{~d}, J=8.0 \mathrm{~Hz}, 1 \mathrm{H}), 8.02-7.98(\mathrm{~m}, 1 \mathrm{H}), 7.96-7.95(\mathrm{~m}, 1 \mathrm{H}), 7.77-7.73(\mathrm{~m}, 1 \mathrm{H}), 7.50-7.48$ $(\mathrm{m}, 2 \mathrm{H}), 7.31(\mathrm{~d}, J=8.0 \mathrm{~Hz}, 2 \mathrm{H}), 2.38(\mathrm{~s}, 3 \mathrm{H}) ;{ }^{13} \mathrm{C}-\mathrm{NMR}$ (DMSO-d6) $\delta 170.6,153.4,147.7,139.7$, 135.7, 134.4, 130.1, 128.4, 128.2, 123.4, 122.9, 122.3, 20.8; HRMS (ESI), $m / z, 253.0798\left[\mathrm{MH}^{+}\right]$, calcd for $\mathrm{C}_{15} \mathrm{H}_{13} \mathrm{~N}_{2} \mathrm{~S}, 253.0799$.

4-(2-Methoxyphenylthio)quinazoline (6). White solid (59\% yield); mp 111-113 ${ }^{\circ} \mathrm{C} ;{ }^{1} \mathrm{H}-\mathrm{NMR}$ (DMSO-d6) $\delta 8.80(\mathrm{~s}, 1 \mathrm{H}), 8.27(\mathrm{~d}, J=8.0 \mathrm{~Hz}, 1 \mathrm{H}), 8.04-8.01(\mathrm{~m}, 1 \mathrm{H}), 7.98-7.96(\mathrm{~m}, 1 \mathrm{H}), 7.80-7.76(\mathrm{~m}, 1 \mathrm{H})$, $7.60-7.54(\mathrm{~m}, 2 \mathrm{H}), 7.20(\mathrm{~d}, J=8.0 \mathrm{~Hz}, 1 \mathrm{H}), 7.10-7.07(\mathrm{~m}, 1 \mathrm{H}), 3.74(\mathrm{~s}, 3 \mathrm{H}) ;{ }^{13} \mathrm{C}-\mathrm{NMR}$ (DMSO-d6) $\delta$ $170.0,160.0,153.5,147.8,137.5,134.4,132.2,128.4,128.2,123.7,122.5,121.2,114.2,112.3,55.9$; HRMS (ESI), $m / z, 269.0747\left[\mathrm{MH}^{+}\right]$, calcd for $\mathrm{C}_{15} \mathrm{H}_{13} \mathrm{~N}_{2} \mathrm{OS}, 269.0749$.

4-(3-Methoxyphenylthio)quinazoline (7). White solid (50\% yield); mp 80-82 ${ }^{\circ} \mathrm{C} ;{ }^{1} \mathrm{H}-\mathrm{NMR}$ (DMSO-d6) $\delta 8.86(\mathrm{~s}, 1 \mathrm{H}), 8.25(\mathrm{~d}, J=8.0 \mathrm{~Hz}, 1 \mathrm{H}), 8.06-8.03(\mathrm{~m}, 1 \mathrm{H}), 8.00-7.98(\mathrm{~m}, 1 \mathrm{H}), 7.81-7.78(\mathrm{~m}, 1 \mathrm{H})$, 7.47-7.44 (m, 1H), 7.24-7.22 (m, 2H), 7.14-7.11 (m, 1H), 3.80 (s, 3H); ${ }^{13} \mathrm{C}-\mathrm{NMR}$ (DMSO-d6) $\delta 170.3$, $159.7,153.5,147.8,134.6,130.3,128.5,128.3,127.8,127.5,123.5,122.4,120.9,115.7$, 55.3; HRMS (ESI), $m / z, 269.0746\left[\mathrm{MH}^{+}\right]$, calcd for $\mathrm{C}_{15} \mathrm{H}_{13} \mathrm{~N}_{2} \mathrm{OS}, 269.0749$.

4-(4-Methoxyphenylthio)quinazoline (8). White solid (86\% yield); mp 125-128 ${ }^{\circ} \mathrm{C} ;{ }^{1} \mathrm{H}-\mathrm{NMR}$ (DMSO-d6) $\delta 8.82(\mathrm{~s}, 1 \mathrm{H}), 8.24(\mathrm{~d}, J=8.0 \mathrm{~Hz}, 1 \mathrm{H}), 8.04-8.01(\mathrm{~m}, 1 \mathrm{H}), 7.98-7.96(\mathrm{~m}, 1 \mathrm{H}), 7.80-7.77(\mathrm{~m}, 1 \mathrm{H})$, 7.57-7.54 (m, 2H), 7.10-7.09 (m, 2H), $3.84(\mathrm{~s}, 3 \mathrm{H}) ;{ }^{13} \mathrm{C}-\mathrm{NMR}$ (DMSO-d6) $\delta 171.1,160.7,153.5$, 147.7, 137.6, 134.6, 128.4, 128.3, 123.6, 122.3, 116.6, 115.3, 55.4; HRMS (ESI), $m / z, 269.0739$ [MH ${ }^{+}$, calcd for $\mathrm{C}_{15} \mathrm{H}_{13} \mathrm{~N}_{2} \mathrm{OS}, 269.0749$.

4-(2-Chlorophenylthio)quinazoline (9). White solid (94\% yield); mp 124-126 ${ }^{\circ} \mathrm{C} ;{ }^{1} \mathrm{H}-\mathrm{NMR}$ (DMSO-d6) $\delta 8.85(\mathrm{~s}, 1 \mathrm{H}), 8.29(\mathrm{~d}, J=8.0 \mathrm{~Hz}, 1 \mathrm{H}), 8.08-8.05(\mathrm{~m}, 1 \mathrm{H}), 8.02-8.00(\mathrm{~m}, 1 \mathrm{H}), 7.84-7.80(\mathrm{~m}, 2 \mathrm{H})$, 7.74-7.72 (m, 1H), 7.62-7.59 (m, 1H), 7.52-7.49 (m, 1H); ${ }^{13} \mathrm{C}-\mathrm{NMR}$ (DMSO-d6) $\delta 168.9,153.4$, $147.9,138.8,138.4,134.7,132.2,130.3,128.51,128.47,128.2,125.9,123.6,122.3$; HRMS (ESI), $m / z$, $273.0244\left[\mathrm{MH}^{+}\right]$, calcd for $\mathrm{C}_{14} \mathrm{H}_{10} \mathrm{ClN}_{2} \mathrm{~S}, 273.0253$. 
4-(3-Chlorophenylthio)quinazoline (10). White solid (66\% yield); mp 125-126 ${ }^{\circ} \mathrm{C} ;{ }^{1} \mathrm{H}-\mathrm{NMR}$ (DMSO-d6) $\delta 8.86(\mathrm{~s}, 1 \mathrm{H}), 8.23(\mathrm{~d}, J=8.0 \mathrm{~Hz}, 1 \mathrm{H}), 8.04-8.03(\mathrm{~m}, 1 \mathrm{H}), 8.00-7.98(\mathrm{~m}, 1 \mathrm{H}), 7.81-7.76(\mathrm{~m}, 2 \mathrm{H})$, 7.65-7.61 (m, 2H), 7.58-7.55 (m, 1H); ${ }^{13} \mathrm{C}-\mathrm{NMR}$ (DMSO-d6) $\delta ~ 169.7, ~ 153.4, ~ 147.9,135.0,134.7$, 134.4, 133.6, 131.1, 129.9, 128.8, 128.5, 128.4, 123.5, 122.3; HRMS (ESI), m/z, $273.0243\left[\mathrm{MH}^{+}\right]$, calcd for $\mathrm{C}_{14} \mathrm{H}_{10} \mathrm{ClN}_{2} \mathrm{~S}, 273.0253$.

4-(4-Chlorophenylthio)quinazoline (11). White solid (91\% yield); mp $133-134{ }^{\circ} \mathrm{C} ;{ }^{1} \mathrm{H}-\mathrm{NMR}$ (DMSO-d6) $\delta 8.85(\mathrm{~s}, 1 \mathrm{H}), 8.24(\mathrm{~d}, J=8.0 \mathrm{~Hz}, 1 \mathrm{H}), 8.07-8.03(\mathrm{~m}, 1 \mathrm{H}), 8.00-7.99(\mathrm{~m}, 1 \mathrm{H}), 7.82-7.79(\mathrm{~m}, 1 \mathrm{H})$,

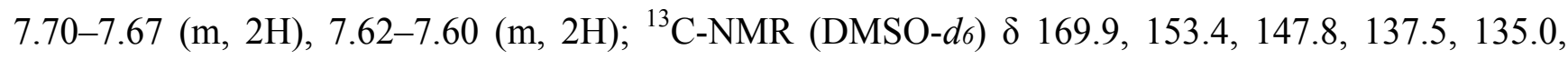
134.7, 129.6, 128.5, 128.4, 125.5, 123.5, 122.3; HRMS (ESI), $m / z, 273.0240\left[\mathrm{MH}^{+}\right]$, calcd for $\mathrm{C}_{14} \mathrm{H}_{10} \mathrm{ClN}_{2} \mathrm{~S}, 273.0253$.

5-Methyl-4-(phenylthio)quinazoline (12). White solid (54\% yield); mp 131-133 ${ }^{\circ} \mathrm{C} ;{ }^{1} \mathrm{H}-\mathrm{NMR}$ (DMSO-d6) $\delta 8.66(\mathrm{~s}, 1 \mathrm{H}), 7.85-7.82(\mathrm{~m}, 1 \mathrm{H}), 7.80-7.78(\mathrm{~m}, 1 \mathrm{H}), 7.62-7.57(\mathrm{~m}, 3 \mathrm{H}), 7.55-7.52(\mathrm{~m}, 3 \mathrm{H}), 3.07$ $(\mathrm{s}, 3 \mathrm{H}) ;{ }^{13} \mathrm{C}-\mathrm{NMR}$ (DMSO-d6) $\delta 171.3,152.2,150.2,135.9,135.4,133.4,130.8,129.7,129.5,128.2$, 127.0, 123.4, 24.7; HRMS (ESI), $m / z, 253.0790\left[\mathrm{MH}^{+}\right]$, calcd for $\mathrm{C}_{15} \mathrm{H}_{13} \mathrm{~N}_{2} \mathrm{~S}, 253.0799$.

6-Methyl-4-(phenylthio) quinazoline (13). White solid (60\% yield); mp 100-102 ${ }^{\circ} \mathrm{C} ;{ }^{1} \mathrm{H}-\mathrm{NMR}$ (DMSO-d6) $\delta 8.83(\mathrm{~s}, 1 \mathrm{H}), 8.00(\mathrm{~s}, 1 \mathrm{H}), 7.89(\mathrm{~d}, J=8.5 \mathrm{~Hz}, 1 \mathrm{H}), 7.73(\mathrm{~d}, J=8.5 \mathrm{~Hz}, 1 \mathrm{H}), 7.67-7.65(\mathrm{~m}, 2 \mathrm{H})$, 7.52-7.51 (m, 3H), $2.62(\mathrm{~s}, 3 \mathrm{H}) ;{ }^{13} \mathrm{C}-\mathrm{NMR}$ (DMSO-d6) $\delta 170.4,153.3,146.9,137.9,136.0,135.8$, 129.7, 129.4, 128.6, 127.4, 123.3, 122.7, 21.8; HRMS (ESI), $m / z, 253.0796\left[\mathrm{MH}^{+}\right]$, calcd for $\mathrm{C}_{15} \mathrm{H}_{13} \mathrm{~N}_{2} \mathrm{~S}, 253.0799$.

8-Methyl-4-(phenylthio)quinazoline (14). White solid (64\% yield); mp 98-100 ${ }^{\circ} \mathrm{C} ;{ }^{1} \mathrm{H}-\mathrm{NMR}$ (DMSO-d6) $\delta 8.84(\mathrm{~s}, 1 \mathrm{H}), 8.04(\mathrm{~d}, J=8.0 \mathrm{~Hz}, 1 \mathrm{H}), 7.85(\mathrm{~d}, J=7.0 \mathrm{~Hz}, 1 \mathrm{H}), 7.65-7.62(\mathrm{~m}, 3 \mathrm{H}), 7.54-7.53(\mathrm{~m}$, $3 \mathrm{H}), 2.66(\mathrm{~s}, 3 \mathrm{H}) ;{ }^{13} \mathrm{C}-\mathrm{NMR}$ (DMSO-d6) $\delta 170.3,152.6,146.8,136.7,135.7,134.2,129.7,129.5$, 127.7, 126.8, 122.2, 121.1, 17.0; HRMS (ESI), $m / z, 253.0789\left[\mathrm{MH}^{+}\right]$, calcd for $\mathrm{C}_{15} \mathrm{H}_{13} \mathrm{~N}_{2} \mathrm{~S}, 253.0799$.

5-Chloro-4-(phenylthio)quinazoline (15). White solid (51\% yield); mp $133-134{ }^{\circ} \mathrm{C} ;{ }^{1} \mathrm{H}-\mathrm{NMR}\left(\mathrm{CDCl}_{3}\right)$ $\delta 8.73(\mathrm{~s}, 1 \mathrm{H}), 7.91-7.89(\mathrm{~m}, 1 \mathrm{H}), 7.74-7.68(\mathrm{~m}, 2 \mathrm{H}), 7.60-7.58(\mathrm{~m}, 2 \mathrm{H}), 7.51-7.50(\mathrm{~m}, 3 \mathrm{H})$; ${ }^{13} \mathrm{C}-\mathrm{NMR}\left(\mathrm{CDCl}_{3}\right) \delta 172.5,153.2,151.3,135.9,132.7,130.6,129.9,129.7,129.4,129.3,128.5,122.6$; HRMS (ESI), $m / z, 273.0244\left[\mathrm{MH}^{+}\right]$, calcd for $\mathrm{C}_{14} \mathrm{H}_{10} \mathrm{ClN}_{2} \mathrm{~S}, 273.0253$.

6-Chloro-4-(phenylthio)quinazoline (16). White solid (79\% yield); mp 103-105 ${ }^{\circ} \mathrm{C} ;{ }^{1} \mathrm{H}-\mathrm{NMR}\left(\mathrm{CDCl}_{3}\right)$ $\delta 8.86(\mathrm{~s}, 1 \mathrm{H}), 8.22(\mathrm{~d}, J=2.0 \mathrm{~Hz}, 1 \mathrm{H}), 7.93(\mathrm{~d}, J=9.0 \mathrm{~Hz}, 1 \mathrm{H}), 7.83-7.81(\mathrm{~m}, 1 \mathrm{H}), 7.66-7.64(\mathrm{~m}$, 2H), 7.53-7.51 (m, 3H); ${ }^{13} \mathrm{C}-\mathrm{NMR}\left(\mathrm{CDCl}_{3}\right) \delta 170.7,154.0,146.9,135.8,134.8,133.3,130.5,130.0$, 129.5, 126.7, 123.8, 122.9; HRMS (ESI), $m / z, 273.0241\left[\mathrm{MH}^{+}\right]$, calcd for $\mathrm{C}_{14} \mathrm{H}_{10} \mathrm{ClN}_{2} \mathrm{~S}, 273.0253$.

7-Chloro-4-(phenylthio)quinazoline (17). White solid (72\% yield); mp 142-143 ${ }^{\circ} \mathrm{C} ;{ }^{1} \mathrm{H}-\mathrm{NMR}$ (DMSO-d6) $\delta 8.85(\mathrm{~s}, 1 \mathrm{H}), 8.29(\mathrm{~d}, J=9.0 \mathrm{~Hz}, 1 \mathrm{H}), 8.07(\mathrm{~d}, J=2.0 \mathrm{~Hz}, 1 \mathrm{H}), 7.83-7.81(\mathrm{~m}, 1 \mathrm{H}), 7.67-7.66(\mathrm{~m}, 2 \mathrm{H})$, 7.57-7.55 (m, 3H); ${ }^{13} \mathrm{C}-\mathrm{NMR}$ (DMSO-d6) $\delta 170.7,154.5,148.6,139.2,135.7,130.0,129.6,128.9$, 127.3, 126.1, 125.7, 121.0; HRMS (ESI), $m / z, 273.0255\left[\mathrm{MH}^{+}\right]$, calcd for $\mathrm{C}_{14} \mathrm{H}_{10} \mathrm{ClN}_{2} \mathrm{~S}, 273.0253$. 
4-Phenoxyquinazoline (18). White solid (75\% yield); mp $123-125{ }^{\circ} \mathrm{C} ;{ }^{1} \mathrm{H}-\mathrm{NMR}\left(\mathrm{CDCl}_{3}\right) \delta 8.80(\mathrm{~s}, 1 \mathrm{H})$, 8.42-8.40 (m, 1H), $8.03(\mathrm{~d}, J=8.5 \mathrm{~Hz}, 1 \mathrm{H}), 7.96-7.94(\mathrm{~m}, 1 \mathrm{H}), 7.69(\mathrm{t}, J=1.0 \mathrm{~Hz}, 1 \mathrm{H}), 7.54-7.50$ $(\mathrm{m}, 2 \mathrm{H}), 7.37-7.35(\mathrm{~m}, 1 \mathrm{H}), 7.30-7.28(\mathrm{~m}, 2 \mathrm{H}) ;{ }^{13} \mathrm{C}-\mathrm{NMR}\left(\mathrm{CDCl}_{3}\right) \delta 167.0,154.4,152.4,151.7,134.2$,

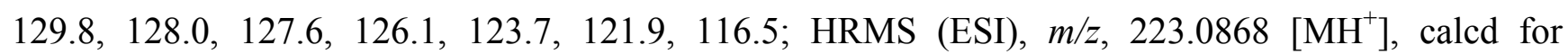
$\mathrm{C}_{14} \mathrm{H}_{11} \mathrm{~N}_{2} \mathrm{O}, 223.0871$.

4-(m-Tolyloxy)quinazoline (19). White solid (52\% yield); mp 100-101 ${ }^{\circ} \mathrm{C} ;{ }^{1} \mathrm{H}-\mathrm{NMR}\left(\mathrm{CDCl}_{3}\right) \delta 8.81$ (s, 1H), 8.41-8.39 (m, 1H), $8.03(\mathrm{~d}, J=8.5 \mathrm{~Hz}, 1 \mathrm{H}), 7.95-7.92(\mathrm{~m}, 1 \mathrm{H}), 7.70-7.67(\mathrm{~m}, 1 \mathrm{H}), 7.41-7.38$ $(\mathrm{d}, J=7.5 \mathrm{~Hz}, 1 \mathrm{H}), 7.17-7.08(\mathrm{~m}, 3 \mathrm{H}), 2.44(\mathrm{~s}, 3 \mathrm{H}) ;{ }^{13} \mathrm{C}-\mathrm{NMR}\left(\mathrm{CDCl}_{3}\right) \delta 167.1,154.4,152.3,151.6$, 140.1, 134.1, 129.5, 127.9, 127.6, 126.9, 123.7, 122.4, 118.8, 116.5, 21.4; HRMS (ESI), m/z, 237.1025 $\left[\mathrm{MH}^{+}\right]$, calcd for $\mathrm{C}_{15} \mathrm{H}_{13} \mathrm{~N}_{2} \mathrm{O}, 237.1028$.

4-(2-Chlorophenoxy)quinazoline (20). White solid (73\% yield); mp $118-119{ }^{\circ} \mathrm{C} ;{ }^{1} \mathrm{H}-\mathrm{NMR}\left(\mathrm{CDCl}_{3}\right) \delta$ $8.78(\mathrm{~s}, 1 \mathrm{H}), 8.46-8.45(\mathrm{~m}, 1 \mathrm{H}), 8.05(\mathrm{~d}, J=8.5 \mathrm{~Hz}, 1 \mathrm{H}), 7.98-7.96(\mathrm{~m}, 1 \mathrm{H}), 7.74-7.72(\mathrm{~m}, 1 \mathrm{H})$, 7.57-7.56 (m, 1H), 7.43-7.41 (m, 1H), 7.38-7.36 (m, 1H), 7.34-7.32 (m, 1H); ${ }^{13} \mathrm{C}-\mathrm{NMR}\left(\mathrm{CDCl}_{3}\right) \delta$ 166.2 , 154.1, 151.9, 148.6, 134.3, 130.7, 128.1, 128.0, 127.8, 127.31, 127.26, 124.1, 123.7, 116.1; HRMS (ESI), $m / z, 257.0479\left[\mathrm{MH}^{+}\right]$, calcd for $\mathrm{C}_{14} \mathrm{H}_{10} \mathrm{ClN}_{2} \mathrm{O}, 257.0482$.

4-(4-Chlorophenoxy)quinazoline (21). White solid (51\% yield); mp $105-108{ }^{\circ} \mathrm{C} ;{ }^{1} \mathrm{H}-\mathrm{NMR}\left(\mathrm{CDCl}_{3}\right) \delta$ $8.79(\mathrm{~s}, \mathrm{H}), 8.39$ (d, $J=8.0 \mathrm{~Hz}, 1 \mathrm{H}), 8.04(\mathrm{~d}, J=8.5 \mathrm{~Hz}, 1 \mathrm{H}), 7.97-7.94(\mathrm{~m}, 1 \mathrm{H}), 7.72-7.69(\mathrm{~m}, 1 \mathrm{H})$, 7.49-7.46 (m, 2H), 7.28-7.23 (m, 2H); ${ }^{13} \mathrm{C}-\mathrm{NMR}\left(\mathrm{CDCl}_{3}\right) \delta 116.8,154.1,151.8,150.9,134.3,131.5$, 129.9, 128.0, 127.8, 123.5, 123.3, 116.3; HRMS (ESI), $m / z, 257.0469\left[\mathrm{MH}^{+}\right]$, calcd for $\mathrm{C}_{14} \mathrm{H}_{10} \mathrm{ClN}_{2} \mathrm{O}$, 257.0482 .

5-Methyl-4-phenoxyquinazoline (22). White solid (53\% yield); mp 73-75 ${ }^{\circ} \mathrm{C} ;{ }^{1} \mathrm{H}-\mathrm{NMR}\left(\mathrm{CDCl}_{3}\right) \delta 8.70$ $(\mathrm{s}, 1 \mathrm{H}), 7.86(\mathrm{~d}, J=8.5 \mathrm{~Hz}, 1 \mathrm{H}), 7.77(\mathrm{t}, J=7.5 \mathrm{~Hz}, 1 \mathrm{H}), 7.53-7.50(\mathrm{~m}, 2 \mathrm{H}), 7.45(\mathrm{~d}, J=7.5 \mathrm{~Hz}, 1 \mathrm{H})$, 7.36-7.33 (m, 1H), 7.28-7.25 (m, 2H), $3.00(\mathrm{~s}, 3 \mathrm{H}) ;{ }^{13} \mathrm{C}-\mathrm{NMR}\left(\mathrm{CDCl}_{3}\right) \delta 168.0,153.7,153.4,152.3$, 136.7, 133.4, 129.9, 129.8, 126.1, 125.9, 122.0, 116.3, 24.1; HRMS (ESI), $m / z, 237.1021\left[\mathrm{MH}^{+}\right]$, calcd for $\mathrm{C}_{15} \mathrm{H}_{13} \mathrm{~N}_{2} \mathrm{O}, 237.1028$.

6-Chloro-4-phenoxyquinazoline (23). White solid (70\% yield); mp 94-97 ${ }^{\circ} \mathrm{C} ;{ }^{1} \mathrm{H}-\mathrm{NMR}\left(\mathrm{CDCl}_{3}\right) \delta 8.77$ (s, 1H), $8.37(\mathrm{~d}, J=2.0 \mathrm{~Hz}, 1 \mathrm{H}), 7.96(\mathrm{~d}, J=9.0 \mathrm{~Hz}, 1 \mathrm{H}), 7.86-7.83(\mathrm{~m}, 1 \mathrm{H}), 7.52-7.49(\mathrm{~m}, 2 \mathrm{H})$, 7.36-7.33 (m, 1H), 7.29-7.27 (m, 2H); ${ }^{13} \mathrm{C}-\mathrm{NMR}\left(\mathrm{CDCl}_{3}\right) \delta 166.1,154.4,152.1,150.1,134.9,133.2$, 129.8, 129.6, 126.2, 122.7, 121.7, 117.1; HRMS (ESI), $m / z, 257.0471\left[\mathrm{MH}^{+}\right]$, calcd for $\mathrm{C}_{14} \mathrm{H}_{10} \mathrm{ClN}_{2} \mathrm{O}$, 257.0482 .

7-Chloro-4-phenoxyquinazoline (24). White solid (70\% yield); mp 190-192 ${ }^{\circ} \mathrm{C} ;{ }^{1} \mathrm{H}-\mathrm{NMR}\left(\mathrm{CDCl}_{3}\right) \delta$ $8.78(\mathrm{~s}, 1 \mathrm{H}), 8.35(\mathrm{~d}, J=8.5 \mathrm{~Hz}, 1 \mathrm{H}), 8.03(\mathrm{~d}, J=2.0 \mathrm{~Hz}, 1 \mathrm{H}), 7.65-7.63(\mathrm{~m}, 1 \mathrm{H}), 7.53-7.50(\mathrm{~m}, 2 \mathrm{H})$, $7.36(\mathrm{t}, J=7.5 \mathrm{~Hz}, 1 \mathrm{H}), 7.29-7.27 \mathrm{ppm}(\mathrm{m}, 2 \mathrm{H}) ;{ }^{13} \mathrm{C}-\mathrm{NMR}\left(\mathrm{CDCl}_{3}\right) \delta 166.9,155.5,152.4,152.2$, $140.5,129.8,128.7,127.2,126.2,125.2,121.8,114.9$; HRMS (ESI), $m / z, 257.0471\left[\mathrm{MH}^{+}\right]$, calcd for $\mathrm{C}_{14} \mathrm{H}_{10} \mathrm{ClN}_{2} \mathrm{O}, 257.0482$.

4-Ethoxyquinazoline (25). White solid (54\% yield); mp 31-33 ${ }^{\circ} \mathrm{C} ;{ }^{1} \mathrm{H}-\mathrm{NMR}\left(\mathrm{CDCl}_{3}\right) \delta 8.80(\mathrm{~s}, 1 \mathrm{H})$, 8.19-8.18 (m, 1H), $7.93(\mathrm{~d}, J=8.0 \mathrm{~Hz}, 1 \mathrm{H}), 7.84-7.81(\mathrm{~m}, 1 \mathrm{H}), 7.58-7.54(\mathrm{~m}, 1 \mathrm{H}), 4.64$ (q, $J=7.0 \mathrm{~Hz}$, 
2H), $1.53(\mathrm{t}, J=7.0 \mathrm{~Hz}, 3 \mathrm{H}) ;{ }^{13} \mathrm{C}-\mathrm{NMR}\left(\mathrm{CDCl}_{3}\right) \delta 166.7,154.5,150.9,133.4,127.7,126.9,123.6$, 116.8, 63.1, 14.4; HRMS (ESI), $m / z, 175.0873\left[\mathrm{MH}^{+}\right]$, calcd for $\mathrm{C}_{10} \mathrm{H}_{11} \mathrm{~N}_{2} \mathrm{O}, 175.0871$.

4-Ethoxy-5-methylquinazoline (26). White solid (33\% yield); mp $66-69{ }^{\circ} \mathrm{C} ;{ }^{1} \mathrm{H}-\mathrm{NMR}\left(\mathrm{CDCl}_{3}\right) \delta 8.72$ (s, 1H), 7.77 (d, $J=8.5 \mathrm{~Hz}, 1 \mathrm{H}), 7.67$ (t, $J=7.5 \mathrm{~Hz}, 1 \mathrm{H}), 7.33$ (d, $J=7.5 \mathrm{~Hz}, 1 \mathrm{H}), 4.61$ (q, $J=7.0 \mathrm{~Hz}$, 2H), $2.86(\mathrm{~s}, 3 \mathrm{H}), 1.54(\mathrm{t}, J=7.0 \mathrm{~Hz}, 3 \mathrm{H}) ;{ }^{13} \mathrm{C}-\mathrm{NMR}\left(\mathrm{CDCl}_{3}\right) \delta 167.8,153.8,152.6,136.9,132.7$, 129.3, 125.8, 116.4, 63.1, 24.0, 14.4; HRMS (ESI), $\mathrm{m} / z, 189.1034\left[\mathrm{MH}^{+}\right]$, calcd for $\mathrm{C}_{11} \mathrm{H}_{13} \mathrm{~N}_{2} \mathrm{O}$, 189.1028.

4-Ethoxy-8-methylquinazoline (27). White solid (67\% yield); mp 31-32 ${ }^{\circ} \mathrm{C} ;{ }^{1} \mathrm{H}-\mathrm{NMR}\left(\mathrm{CDCl}_{3}\right) \delta 8.84$ $(\mathrm{s}, 1 \mathrm{H}), 8.04-8.02(\mathrm{~m}, 1 \mathrm{H}), 7.66(\mathrm{~d}, J=7.5 \mathrm{~Hz}, 1 \mathrm{H}), 7.44(\mathrm{t}, J=8.0 \mathrm{~Hz}, 1 \mathrm{H}), 4.63(\mathrm{q}, J=7.0 \mathrm{~Hz}, 2 \mathrm{H})$, $2.73(\mathrm{~s}, 3 \mathrm{H}), 1.52(\mathrm{t}, J=7.0 \mathrm{~Hz}, 3 \mathrm{H}) ;{ }^{13} \mathrm{C}-\mathrm{NMR}\left(\mathrm{CDCl}_{3}\right) \delta 166.9,153.4,150.0,135.9,133.5,126.4$, 121.2, 116.6, 62.9, 17.6, 14.3; HRMS (ESI), $m / z, 189.1023\left[\mathrm{MH}^{+}\right]$, calcd for $\mathrm{C}_{11} \mathrm{H}_{13} \mathrm{~N}_{2} \mathrm{O}, 189.1028$.

7-Chloro-4-ethoxyquinazoline (28). White solid (64\% yield); mp $87-88{ }^{\circ} \mathrm{C} ;{ }^{1} \mathrm{H}-\mathrm{NMR}\left(\mathrm{CDCl}_{3}\right) \delta 8.76$ $(\mathrm{s}, 1 \mathrm{H}), 8.08(\mathrm{~d}, J=9.0 \mathrm{~Hz}, 1 \mathrm{H}), 7.90(\mathrm{~d}, J=1.5 \mathrm{~Hz}, 1 \mathrm{H}), 7.49-7.47(\mathrm{~m}, 1 \mathrm{H}), 4.62(\mathrm{q}, J=7.0 \mathrm{~Hz}, 2 \mathrm{H})$, $1.51(\mathrm{t}, J=7.0 \mathrm{~Hz}, 3 \mathrm{H}) ;{ }^{13} \mathrm{C}-\mathrm{NMR}\left(\mathrm{CDCl}_{3}\right) \delta 166.6,155.5,151.6,139.6,127.8,126.9,125.0,115.0$, 63.3, 14.3; HRMS (ESI), $m / z, 209.0479\left[\mathrm{MH}^{+}\right]$, calcd for $\mathrm{C}_{10} \mathrm{H}_{10} \mathrm{ClN}_{2} \mathrm{O}, 209.0482$.

4-Propoxyquinazoline (29). White solid (48\% yield); mp 190-192 ${ }^{\circ} \mathrm{C} ;{ }^{1} \mathrm{H}-\mathrm{NMR}\left(\mathrm{CDCl}_{3}\right) \delta 8.79(\mathrm{~s}, 1 \mathrm{H})$, 8.19-8.17 (m, 1H), $7.92(\mathrm{~d}, J=8.5 \mathrm{~Hz}, 1 \mathrm{H}), 7.83-7.80(\mathrm{~m}, 1 \mathrm{H}), 7.57-7.53(\mathrm{~m}, 1 \mathrm{H}), 4.52(\mathrm{t}, J=6.5 \mathrm{~Hz}$, 2H), $1.95-1.91(\mathrm{~m}, 2 \mathrm{H}), 1.10(\mathrm{t}, J=7.5 \mathrm{~Hz}, 3 \mathrm{H}) ;{ }^{13} \mathrm{C}-\mathrm{NMR}\left(\mathrm{CDCl}_{3}\right) \delta 166.8,154.4,150.9,133.4$, 127.6, 126.9, 123.5, 116.7, 68.7, 22.1, 10.5; HRMS (ESI), $m / z, 189.1023\left[\mathrm{MH}^{+}\right]$, calcd for $\mathrm{C}_{11} \mathrm{H}_{13} \mathrm{~N}_{2} \mathrm{O}$, 189.1028 .

\section{Conclusions}

In summary, we have successfully developed an HCCP-mediated direct formation of thioethers (4-arylthioquinazolines) from quinazolin-4(3H)-ones and thiophenols in moderate to excellent yields. This method has also been utilized to prepare quinazoline ethers, including 4-aryloxyquinazolines and 4-alkoxyquinazolines, using phenols and sodium alkoxides as the nucleophiles. This direct formation of quinazoline (thio)ethers is mild, convenient, and suitable for a wide range of less expensive nucleophiles. This methodology would facilitate the syntheses of 4-arylthioquinazoline and 4-aryloxyquinazoline derivatives in medicinal chemistry.

\section{Acknowledgments}

The authors gratefully acknowledge the financial support of the National Natural Science Foundations of China (NSFC 21146001), and the Key Science and Technology Innovation Team of Zhejiang Province (2009R09002-04).

\section{Conflict of Interest}

The authors declare no conflict of interest. 


\section{References}

1. Hori, M.; Iemura, R.; Hara, H.; Ozaki, A.; Sukamoto, T.; Ohtaka, H. Novel 4-phenoxy-2-(1piperazinyl)quinazolines as potent anticonvulsive and antihypoxic agents. Chem. Pharm. Bull. 1990, 38, 681-687.

2. Havera, H.J.; Vidrio, H.J. Derivatives of 1,3-disubstituted 2,4(1H,3H)-quinazolinediones as possible peripheral vasodilators or antihypertensive agents. J. Med. Chem. 1979, 22, 1548-1550.

3. Chao, Q.; Deng, L.; Shih, H.; Leoni, L.M.; Genini, D.; Carson, D.A.; Cottam, H.B. Substituted isoquinolines and quinazolines as potential antiinflammatory agents. Synthesis and biological evaluation of inhibitors of tumor necrosis factor $\alpha . J$. Med. Chem. 1999, 42, 3860-3873.

4. Kung, P.P.; Casper, M.D.; Cook, K.L.; Wilson-Lingardo, L.; Risen, L.M.; Vickers, T.A.; Ranken, R.; Blyn, L.B.; Wyatt, J.R.; Cook, P.D.; et al. Structure-activity relationships of novel 2-substituted quinazoline antibacterial agents. J. Med. Chem. 1999, 42, 4705-4713.

5. Liverton, N.J.; Armstrong, D.J.; Claremon, D.A.; Remy, D.C.; Bardwin, J.J.; Lynch, R.J.; Zhang, G.; Gould, R.J. Nonpeptide glycoprotein IIb/IIIa inhibitors: Substituted quinazolinediones and quinazolinones as potent fibrinogen receptor antagonists. Bioorg. Med. Chem. Lett. 1998, 8, 483-486.

6. Brunton, S.A.; Stibbard, J.H.A.; Rubin, L.L.; Kruse, L.I.; Guicherit, O.M.; Boyd, E.A.; Price, S. Potent inhibitors of the Hedgehog signaling pathway. J. Med. Chem. 2008, 51, 1108-1110.

7. Smits, R.A.; de Esch, I.J.P.; Zuiderveld, O.P.; Broeker, J.; Sansuk, K.; Guaita, E.; Coruzzi, G.; Adami, M.; Haaksma, E.; Leurs, R. Discovery of quinazolines as histamine $\mathrm{H}_{4}$ receptor inverse agonists using a scaffold hopping approach. J. Med. Chem. 2008, 51, 7855-7865.

8. Sirisoma, N; Pervin, A.; Zhang, H.; Jiang, S.C.; Willardsen, J.A.; Anderson, M.B.; Mather, G.; Pleiman,C.M.; Kasibhatla, S.; Tseng, B.; et al. Discovery of $N$-(4-methoxyphenyl)-N,2dimethylquinazolin-4-amine, a potent apoptosis inducer and efficacious anticancer agent with high blood brain barrier penetration. J. Med. Chem. 2009, 52, 2341-2351.

9. Zeng, Z.-S.; He, Q.-Q.; Liang, Y.-H.; Feng, X.-Q.; Chen, F.-E.; Clercq, E.D.; Balzarini, J. Hybrid diarylbenzopyrimidine non-nucleoside reverse transcriptase inhibitors as promising new leads for improved anti-HIV-1 chemotherapy. Bioorg. Med. Chem. 2010, 18, 5039-5047.

10. Nakamura, H.; Onagi, S. Synthesis and biological evaluation of allenic quinazolines using palladium-catalyzed hydride-transfer reaction. Tetrahedron Lett. 2006, 47, 2539-2542.

11. Castera-Ducros, C.; Azas, N.; Verhaeghe, P.; Hutter, S.; Garrigue, P.; Dumetre, A.; Mbatchi, L.; Laget, M.; Remusat, V.; Sifredi, F.; et al. Targeting the human malaria parasite Plasmodium falciparum: In vitro identification of a new antiplasmodial hit in 4-phenoxy-2trichloromethylquinazoline series. Eur. J. Med. Chem. 2011, 46, 4184-4191.

12. Holladay, M.W.; Campbell, B.T.; Rowbottom, M.W.; Chao, Q.; Sprankle, K.G.; Lai, A.G.; Abraham, S.; Setti, E.; Faraoni, R.; Tran, L.; et al. 4-Quinazolinyloxy-diaryl ureas as novel BRAF $^{\mathrm{V} 600 \mathrm{E}}$ inhibitors. Bioorg. Med. Chem. Lett. 2011, 21, 5342-5346.

13. Rowbottom, M.W.; Faraoni, R.; Chao, Q.; Campbell, B.T.; Lai, A.G.; Setti, E.; Ezawa, M.; Sprankle, K.G.; Abraham, S.; Tran, L.; et al. Identification of 1-(3-(6,7-dimethoxyquinazolin-4yloxy)phenyl)-3-(5-(1,1,1-trifluoro-2-methylpropan-2-yl)isoxazol-3-yl)urea hydrochloride (CEP-32496), a highly potent and orally efficacious inhibitor of V-RAF murine sarcoma viral oncogene homologue B1 (BRAF) V600E. J. Med. Chem. 2012, 55, 1082-1105. 
14. Ple, P.A.; Jung, F.; Ashton, S.; Hennequin, L.; Laine, R.; Morgentin, R.; Pasquet, G.; Taylor, S. Discovery of AZD2932, a new Quinazoline Ether Inhibitor with high affinity for VEGFR-2 and PDGFR tyrosine kinases. Bioorg. Med. Chem. Lett. 2012, 22, 262-266.

15. Yang, S.; Li, Z.; Jin, L.H.; Song, B.A.; Liu, G.; Chen, J.; Chen, Z.; Hu, D.Y.; Xue, W.; Xu, R.Q. Synthesis and bioactivity of 4-alkyl(aryl)thioquinazoline derivatives. Bioorg. Med. Chem. Lett. 2007, 17, 2193-2196.

16. Verhaeghe, P.; Dumetre, A.; Castera-Ducros, C.; Hutter, S.; Laget, M.; Fersing, C.; Prieri, M.; Yzombard, J.; Sifredi, F.; Rault, S.; et al. 4-Thiophenoxy-2-trichloromethyquinazolines display in vitro selective antiplasmodial activity against the human malaria parasite Plasmodium falciparum. Bioorg. Med. Chem. Lett. 2011, 21, 6003-6006.

17. Garofalo, A.; Goossens, L.; Baldeyrou, B.; Lemoine, A.; Ravez, S.; Six, P.; David-Cordonnier, M.-H.; Bonte, J.-P.; Depreux, P.; Lansiaux, A.; et al. Design, synthesis, and DNA-binding of $\mathrm{N}$-alkyl(anilino)quinazoline derivatives. J. Med. Chem. 2010, 53, 8089-8103.

18. Harris, C.S.; Hennequin, L.F.; Willerval, O. Three-point variation of a gefinitib quinazoline core. Tetrahedron Lett. 2009, 50, 1600-1602.

19. Sreedhar, B.; Reddy, P.S.; Reddy, M.A. Catalyst-free and base-free water-promoted SNAr reaction of heteroaryl halides with thiols. Synthesis 2009, 1732-1738.

20. Wan, Z.K.; Wacharasindhu, S.; Binnun, E.; Mansour, T. An efficient direct amination of cyclic amides and cyclic ureas. Org. Lett. 2006, 8, 2425-2428.

21. Wan, Z.K.; Wacharasindhu, S.; Levins, C.G.; Lin, M.; Tabei, K.; Mansour, T.S. The scope and mechanism of phosphonium-mediated $\mathrm{S}_{\mathrm{N}} \mathrm{Ar}$ reactions in heterocyclic amidesand ureas. J. Org. Chem. 2007, 72, 10194-10210.

22. Shen, Z.L.; Hong, Y.M.; He, X.F.; Mo, W.M.; Hu, B.X.; Sun, N.; Hu, X.Q. Switching the chemoselectivity in the amination of 4-chloroquinazolines with aminopyrazoles. Org. Lett. 2010, $12,552-555$.

23. Shen, Z.L.; He, X.F.; Hong, Y.M.; Hu, X.Q.; Mo, W.M.; Hu, B.X.; Sun, N. One-pot synthesis of 4-aminoquinazolines by hexamethyldisilazane-mediated reaction of quinazolin-4(3H)-ones with amines. Synth. Commun. 2011, 41, 3644-3653.

24. Shen, Z.L.; He, X.F.; Dai, J.L.; Mo, W.M.; Hu, B.X.; Sun, N.; Hu, X.Q. An efficient HCCP-mediated direct amination of quinazolin-4(3H)-ones. Tetrahedron 2011, 67, 1665-1672.

Sample Availability: Samples of the compounds 3-17 are available from the authors.

(C) 2013 by the authors; licensee MDPI, Basel, Switzerland. This article is an open access article distributed under the terms and conditions of the Creative Commons Attribution license (http://creativecommons.org/licenses/by/3.0/). 